\title{
R-adaptation de maillage par l'estimateur d'erreur hiérarchique
}

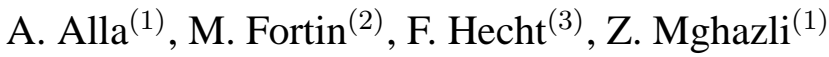 \\ (1) Laboratoire SIANO, Université Ibn Tofail, Faculté des Siences, B.P. 133, Kénitra, Maroc. \\ (2) GIREF, Université Laval, Québec, G1K-7P4, Canada. \\ (3) Laboratoire J.L. Lions, Université Pierre et Marie Curie, B.C. 187, 4 place Jussieu, Paris Cedex \\ 05, France.
}

RÉSUMÉ. L'objectif de ce travail est de déterminer la meilleure position des noeuds d'un maillage, utilisé lors de la discrétisation d'un problème aux limites par la méthode des éléments finis. La procédure de déplacement des nœuds (appelé aussi R-adaptation) est une étape importante dans la stratégie globale d'adaptation de maillage. La position optimale des nœuds est déterminée en minimisant l'erreur d'approximation. Pour évaluer cette erreur nous utilisons l'estimateur d'erreur hiérarchique. Un test numérique est présenté.

ABSTRACT. The ojective of this work is to devise a method to determine the optimal position of the nodes in a finite element discretization for a boundary value problem. The node displacement procedure (also called R-adaptation) is a crucial step in a global mesh adaptation procedure. In the present approch, we determine the nodal position by minimizing the approximation error. This error is evaluated using a hierarchical estimator. A numerical test is presented

MOTS-CLÉS : méthode des éléments finis, adaptation de maillage, estimateur hiérarchique.

KEYWORDS : Finite element method, mesh adaptation, hierarchical estimator. 


\section{Introduction}

Les estimations a posteriori et les procédures d'adaptation de maillage sont devenues ces dernières décennies, l'un des principaux axes de développement dans le domaine de l'analyse numérique et un outil indispensable dans la discrétisation des équations aux dérivées partielles. Elles ont pour but d'améliorer la qualité de la solution calculée et permettent d'assurer, d'une part que la solution approchée est d'une précision fixée, et d'autre part que cette précision est equidistribuée.

Les estimations a posteriori d'erreur sont données en termes d'indicateurs d'erreur qui peuvent être locaux et qui ne tiennent compte que de la solution approchée $u_{h}$ effectivement calculée et des donnés du problème. Elles permettent d'identifier les régions du domaine source d'erreurs grossières. Plusieurs type d' estimations a posteriori ont été développés depuis les travaux pionniers de Babushka et Rheinboldt [2],[3]. On en distingue les estimations par résidus, les estimations par interpolation, les estimations par résolution de problèmes locaux, les estimations par dualité, les estimations hiérarchiques ([4],[5], [1]), ... Une description générale des estimations a posteriori pour les problèmes elliptiques se trouve dans [9].

La méthode hiérarchique est basée sur une idée simple : étant donné une approximation par éléments finis d'ordre $k$, une meilleure approximation, d'ordre $k+1$, peut être utilisée pour estimer la précision de la solution calculée. Pour éviter de procéder à des résolutions couteuses on utilise une base hiérarchique de l'espace d'approximation éléments finis ([5]).

Ayant obtenu un estimateur d'erreur a posteriori, ce dernier est utilisé comme critère pour construire un procédé d'adaptation de maillage de manière à amener l'erreur réellement commise à un niveau choisi par l'utilisateur. Les algorithmes d'adaptation sont généralement basés sur des procédés de remaillage par création de sommets (raffinement), retournement d'arêtes, suppression de sommets (déraffinement) et déplacement des nœuds. Dans ce travail, nous nous intéressons aux déplacements des nœuds du maillage, et cherchons la position optimale, dans le sens qui réduit l'erreur d'approximation $u-u_{h}$, pour un problème linéaire elliptique de second ordre discrétisé par une méthodes éléments finis conformes. Mathématiquement, ce problème est équivalent à un problème de minimisation d'une fonctionnelle coût liée à l'erreur d'approximation. Une étude dans ce sens a été réalisée dans [6] en utilisant la fonctionnelle d'énérgie comme fonction coût. Nous nous proposons ici d'utiliser la norme de l'estimateur hiérarchique comme fonctionnelle coût sous la contrainte de deux problèmes variationnels discrets, l'un lié à la solution approchée $u_{h}$ et l'autre lié à l'estimateur d'erreur hiérarchique. Ceci pourra constituer une justification mathématique de la procédure de déplacement des nœuds qui est souvent opérée, dans les différents codes de calcul, d'une manière intuitive. Le problème ainsi posé, est un problème d'optimisation de forme dont les paramètres sont la position des 
nœuds du maillage. Nous utlisons les techniques de [10],[11],[8] pour calculer la dérivée de forme de la fonctionnelle coût.

\section{Estimateur d'erreur hiérarchique}

Etant donné un ouvert polygonal $\Omega$ de $\mathbb{R}^{n}$, de frontière $\Gamma=\partial \Omega$, considérons le problème modèle

$$
\left\{\begin{array}{l}
\text { trouver } u \in \mathcal{V}, \text { tel que : } \\
a(u, v)=(F, v) \quad \forall v \in \mathcal{V}
\end{array}\right.
$$

où $a(u, v):=\int_{\Omega} \nabla u \cdot \nabla v d x$, l'espace $\mathcal{V}=H_{0}^{1}(\Omega)$ muni de la norme $\left\||v \||^{2}=a(v, v)\right.$, et $F$ un élément de $L^{2}(\Omega)$ muni du produit scalaire $(\cdot, \cdot)$. Nous approchons le problème (1) par une méthode d'éléments finis. Si $\mathcal{T}_{h}$ est une triangulation régulière de $\Omega$, on considère l'espace éléments finis de degrés $k, \mathcal{V}_{h}^{(k)}$, défini par

$$
\mathcal{V}_{h}^{(k)}=\left\{v_{h} \in \mathcal{V} ; v_{h \mid K} \in P_{k}(K), \forall K \in \mathcal{T}_{h}\right\},
$$

où $P_{k}(K)$ est l'espace des polynômes de degré $\leq k$ dans $K$.

le problème approché est donné par

$$
\left\{\begin{array}{l}
\text { trouver } u_{h}^{(k)} \in \mathcal{V}_{h}^{(k)}, \text { tel que : } \\
a\left(u_{h}^{(k)}, v_{h}\right)=\left(F, v_{h}\right) \quad \forall v_{h} \in \mathcal{V}_{h}^{(k)} .
\end{array}\right.
$$

L'erreur $e=u-u_{h}^{(k)}$, commise en approchant (1) par (2) est solution du problème

$$
a(e, w)=r(w) \quad \forall w \in V
$$

où $r(w)=(F, w)-a\left(u_{h}^{(k)}, w\right)$ est le résidu de l' équation (2). Une approximation de cette erreur peut être obtenue en considérant $\mathcal{V}_{h}^{(k+1)}$, l'espace éléments finis de degré $k+1$ et en résolvant dans $\mathcal{V}_{h}^{(k+1)}$ le problème

$$
a\left(e_{h}^{(k+1)}, w_{h}\right)=r\left(w_{h}\right) \quad \forall w_{h} \in \mathcal{V}_{h}^{(k+1)} .
$$

Ceci revient à résoudre le problème au complet dans $\mathcal{V}_{h}^{(k+1)}$, ce qui peut être très couteux. L'idée ici (cf. [5]) est d'utiliser une base hiérarchique de l'espace d'approximation $\mathcal{V}_{h}^{(k+1)}$ de manière à ce que ce dernier soit la somme directe de $\mathcal{V}_{h}^{(k)}$ (polynôme de degré $k$ ) et d'un espace de correction $E_{h}$ de polynômes de degré $k+1$

$$
\mathcal{V}_{h}^{(k+1)}=\mathcal{V}_{h}^{(k)} \oplus E_{h}
$$


Nous considérons ici le cas $k=1$. L'approximation linéaire par morceaux est alors corrigée par une approximation quadratique par morceaux où seulement les nœuds des milieux des côtés sont calculés, les valeurs des sommets restant inchangées. L'erreur peut donc être approchée par la résolution du problème

$$
\left\{\begin{array}{l}
\text { trouver } e_{h} \in E_{h}, \text { tel que : } \\
a\left(e_{h}, \hat{w}_{h}\right)=r\left(\hat{w}_{h}\right) \quad \forall \hat{w}_{h} \in E_{h} .
\end{array}\right.
$$

Nous résolvons donc un problème global mais dont la dimension, qui est celle de l'espace $E_{h}$, est beaucoup plus petite que celle de $\mathcal{V}_{h}^{(2)}$. Ce problème est bien conditionné (cf.[9]) et peut être résolu en quelques itérations par la méthode du gradient conjugué par exemple.

La méthode hiérarchique a été analysée pour une large classe de problèmes aux limites et le résultat principal est global.

Théorème 1 (voir [5]) Sous les hypothèses

1) Hypothèse de saturation

$$
\exists \beta, \quad 0<\beta<1 \text { tel que }\left\|\left|u-u_{h}^{(k+1)}\left\|\left|\leq \beta\left\|\left|u-u_{h}^{(k)} \|\right|\right.\right.\right.\right.\right.
$$

2) Inégalité de Cauchy-Binyakowski-Schwarz

$$
\left\{\begin{array}{l}
\exists \gamma<1 \quad \text { indépendant de } h, \text { tel que } \\
\forall v \in \mathcal{V}_{h}^{(k)} \quad \forall w \in E_{h}|a(v, w)|<\gamma\||v\||\||w \||,
\end{array}\right.
$$

nous avons

$$
\left.\left(1-\beta^{2}\right)\left(1-\gamma^{2}\right)\left\|\left|u-u_{h}^{(k)}\left\|\left.\right|^{2} \leq\right\|\right| e_{h}\right\|\right|^{2} \leq\left\|\left|u-u_{h}^{(k)} \|\right|^{2} .\right.
$$

\section{Position du problème d'optimisation de forme}

Etant la solution du problème (3) dont le second membre dépend des données et de la solution $u_{h}$, l'estimateur hiérarchique $e_{h}$, est une quantité calculable, équivalente à l'erreur $e=u-u_{h}$ dans le sens de l'inégalité (4). Il peut donc être utilisé comme critère dans une stratégie d'adaptation du maillage, qui comporte plusieurs opérations : retournement d'arrêtes, création de nœuds, suppression de nœuds et déplacement de nœuds. Nous proposons dans ce travail une méthode permettant de déterminer la position optimale des nœuds du maillage dans la résolution du problème (2), en utilisant l'estimateur hiérarchique. Pour un maillage donné $\mathcal{T}_{h}$, les solutions des problèmes (2) et (3) qui, bien entendu, dépendent de $\mathcal{T}_{h}$, seront notées respectivement $u_{h}\left(\mathcal{T}_{h}\right)$ et $e_{h}\left(\mathcal{T}_{h}\right)$.

Considérons la fonctionnelle

$$
J\left(e_{h}\left(\mathcal{T}_{h}\right)\right):=\frac{1}{2} \int_{\Omega}\left|\nabla e_{h}\left(\mathcal{T}_{h}\right)\right|^{2}=\frac{1}{2}\left|\left\|e_{h}\left(\mathcal{T}_{h}\right) \mid\right\|^{2} .\right.
$$


Le problème est donc le suivant

$$
\left\{\begin{array}{l}
\text { Trouver la position des nœuds de } \mathcal{T}_{h} \text { qui minimise } J\left(e_{h}\left(\mathcal{T}_{h}\right)\right), \\
\text { sous la contrainte } \\
\left\{\begin{array}{l}
a\left(e_{h}, \hat{v}_{h}\right)=\left(F, \hat{v}_{h}\right)-a\left(u_{h}, \hat{v}_{h}\right) \\
a\left(u_{h}, v_{h}\right)=\left(F, v_{h}\right) \quad \forall v_{h} \in \mathcal{V}_{h} .
\end{array} \quad \forall \hat{v}_{h} \in E_{h}\right.
\end{array}\right.
$$

Notons $\mathcal{M}$ l'ensemble des nœuds de $\mathcal{T}_{h}$ et $\mathcal{T}_{T}$ la famille des triangulations $\mathcal{T}_{h}(\mathcal{M})$ engendrées par les $\mathcal{M}$ ayant une même table topologique, et formant l'ensemble $m_{T}$. Le problème d'optimisation de forme qu'on considère est donc le suivant.

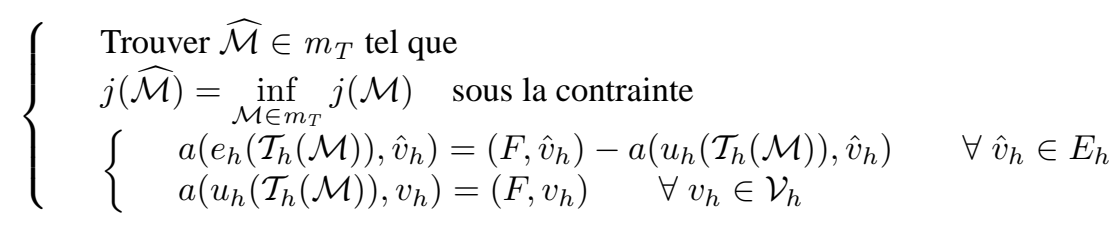

où $j(\mathcal{M}):=J\left(e_{h}\left(\mathcal{T}_{h}(\mathcal{M})\right)\right.$.

\section{Algorithme}

Nous supposons que les problèmes (5) et (6) admettent une unique solution. Pour calculer la dérivée de la fonctionnelle $j(\cdot)$ par rapport aux paramètres de forme qui sont les nœuds du maillage, nous introduisons le Lagrangien pour transformer le problème avec contraintes (5), en un problème sans contraintes. Ce Lagrangien dans $E_{h} \times \mathcal{V}_{h}^{(1)} \times$ $E_{h} \times \mathcal{V}_{h}^{(1)}$ est donné par

$\mathcal{L}\left(\hat{v}_{h}, v_{h}, q_{h}, \mu_{h}\right)=\frac{1}{2}\left\|\hat{v}_{h}\right\|^{2}+a\left(\hat{v}_{h}, q_{h}\right)-\left(F, q_{h}\right)+a\left(v_{h}, q_{h}\right)+a\left(v_{h}, \mu_{h}\right)-\left(F, \mu_{h}\right)$.

Les deux problèmes adjoints associés sont donnés par

$$
\begin{aligned}
& \left\{\begin{array}{l}
\text { trouver } p_{h} \in E_{h}, \quad \text { telle que : } \\
\int_{\Omega_{h}} \nabla p_{h} \nabla \hat{v}_{h} d \Omega_{h}=-\int_{\Omega_{h}} \nabla e_{h} \nabla \hat{v}_{h} d \Omega_{h} \quad \forall \hat{v}_{h} \in E_{h} .
\end{array}\right. \\
& \left\{\begin{array}{l}
\text { trouver } \lambda_{h} \in \mathcal{V}_{h}, \quad \text { telle que : } \\
\int_{\Omega_{h}} \nabla \lambda_{h} \nabla v_{h} d \Omega_{h}=-\int_{\Omega_{h}} \nabla p_{h} \nabla v_{h} d \Omega_{h}
\end{array} \quad \forall v_{h} \in \mathcal{V}_{h},\right.
\end{aligned}
$$

où la notation $\Omega_{h}$ signifie que le domaine $\Omega$ est maillé avec la triangulation $\mathcal{T}_{h}$.

Pour calculer la dérivée de $j(\cdot)$ par rapport au domaine on utilise les techniques de [6], [11] et [8]. Soit $T_{t}$ une transformation donnée par un champ de vitesse $V$, telle que 
$T_{t}\left(\Omega_{h}\right)=\Omega_{h}^{t}$. On note $e_{h}^{t}:=e_{h} \circ T_{t}^{-1}$ et $\mathcal{M}^{t}=T_{t}(\mathcal{M})$.

A l'instant $t>0$, nous avons par changement de variable

$$
j\left(\mathcal{M}^{t}\right)=\frac{1}{2} \int_{\Omega_{h}^{t}}\left|\nabla e_{h}^{t}\right|^{2} d \Omega_{h}^{t}=\frac{1}{2} \int_{\Omega_{h}} A(t) \nabla e_{h} . \nabla e_{h} d \Omega_{h},
$$

où $A(t)=D T_{t}^{-1} J_{t}^{*} D T_{t}^{-1}$ et $J_{t}=\operatorname{det}\left(D T_{t}\right)$. La dérivation de $j\left(\mathcal{M}^{t}\right)$ par rapport à $t$ à l'instant $t=0$ donne

$$
d j\left(\mathcal{M}^{t} ; V\right)=\frac{1}{2} \int_{\Omega_{h}} A^{\prime}(0) \nabla e_{h} \cdot \nabla e_{h} d \Omega_{h} \quad+\int_{\Omega_{h}} \nabla e_{h} \nabla \dot{e}_{h} d \Omega_{h} .
$$

À l'instant $t>0$, le problème (3) est donné par

$$
\int_{\Omega_{h}^{t}} \nabla e_{h}^{t} \nabla \hat{w}_{h} d \Omega_{h}^{t}=\int_{\Omega_{h}^{t}} F \hat{w}_{h} d \Omega_{h}^{t}-\int_{\Omega_{h}^{t}} \nabla u_{h}^{t} \nabla \hat{w}_{h} d \Omega_{h}^{t}
$$

L'application d' un changement de variable donne

$$
\int_{\Omega_{h}} A(t) \nabla e_{h} \cdot \nabla \hat{w}_{h} d \Omega_{h}=\int_{\Omega_{h}} J_{t} F \circ T_{t} \hat{w}_{h} d \Omega_{h}-\int_{\Omega_{h}} A(t) \nabla u_{h} . \nabla \hat{w}_{h} d \Omega_{h}
$$

On utilise la dérivée des formes bilinéaires, en posant $\hat{w}_{h}=p_{h}$, nous obtenons

$$
\begin{aligned}
\int_{\Omega_{h}} \nabla \dot{e}_{h} \nabla p_{h} d \Omega_{h}= & \int_{\Omega_{h}} \operatorname{div}(F V(0)) p_{h} d \Omega_{h}-\int_{\Omega_{h}} A^{\prime}(0) \nabla\left(e_{h}+u_{h}\right) \cdot \nabla p_{h} d \Omega_{h} \\
& -\int_{\Omega_{h}} \nabla \dot{u}_{h} \nabla p_{h} d \Omega_{h} .
\end{aligned}
$$

Dans le problème (7), on pose $\hat{v}_{h}=\dot{e}_{h}$ pour obtenir

$$
\begin{aligned}
d j\left(\mathcal{M}^{t} ; V\right)= & \frac{1}{2} \int_{\Omega_{h}} A^{\prime}(0) \nabla e_{h} \cdot \nabla e_{h} d \Omega_{h}+\int_{\Omega} A^{\prime}(0)\left(\nabla u_{h}+\nabla e_{h}\right) \cdot \nabla p_{h} d \Omega_{h} \\
& -\int_{\Omega_{h}} \operatorname{div}(F V(0)) p_{h} d \Omega_{h}+\int_{\Omega_{h}} \nabla \dot{u}_{h} \nabla p_{h} d \Omega_{h} .
\end{aligned}
$$

De même, on applique un changement de variable au problème (2) donné à un instant $t>0$, puis on utilise la dérivée des formes bilinéaires en posant $v_{h}=\lambda_{h}$ dans la formule obtenue et $\hat{v}_{h}=\dot{u}_{h}$ dans le problème (8), on obtient finalement une écriture explicite de la dérivée de $j(\cdot)$, donnée par

$$
\begin{aligned}
d j\left(\mathcal{M}^{t} ; V\right)= & \frac{1}{2} \int_{\Omega_{h}} A^{\prime}(0) \nabla e_{h} . \nabla e_{h} d \Omega_{h}-\int_{\Omega_{h}} \operatorname{div}(F V(0))\left(p_{h}+\lambda_{h}\right) d \Omega_{h} \\
& +\int_{\Omega_{h}} A^{\prime}(0) \nabla u_{h} . \nabla \lambda_{h} d \Omega_{h}+\int_{\Omega_{h}} A^{\prime}(0) \nabla\left(u_{h}+e_{h}\right) . \nabla p_{h} d \Omega_{h} .
\end{aligned}
$$


Soit $\left\{e_{i}\right\}_{i}$ une base de $\mathcal{V}_{h}^{(1)}$, un choix particulier (cf. ([6],[10]) de champ de vitesse $V=\left(e_{i}, 0\right)$ dans la formule de la dérivée, permet de calculer la dérivée partielle de $j(\cdot)$ par rapport à la première variable $x_{i}^{1}$. De même le choix $V=\left(0, e_{i}\right)$ permet de calculer la dérivée partielle par rapport à la deuxième variable $x_{i}^{2}$.

L'algorithme utilisé pour le déplacement des nœuds est décrit comme suit.

Données : La précision $\varepsilon=\varepsilon_{0}$, la triangulation initiale $\mathcal{T}_{h}^{(0)}$.

Etape 1 : Résolution du problème (2), (calcul de $u_{h}$ ).

Etape 2 : Résolution du problème (3), (calcul de $e_{h}$, calcul de $j$ ).

Etape 3 : Résolution des problèmes $(7)$ et $(8)$, (calcul de $p_{h}$ et $\lambda_{h}$ ).

Etape 4 : Pour chaque nœud faire

- calcul de $d j\left(\mathcal{M}, V_{1}\right)$ et de $d j\left(\mathcal{M}, V_{2}\right)$ où $V_{1}=\left(e_{i}, 0\right)$ et $V_{2}=\left(0, e_{i}\right)$;

- calcul de $\|d j(\mathcal{M}, V)\|=\left(\left(\operatorname{dj}\left(\mathcal{M}, V_{1}\right)\right)^{2}+\left(\operatorname{dj}\left(\mathcal{M}, V_{2}\right)\right)^{2}\right)^{\frac{1}{2}}$.

Etape 5 : On pose test $:=$ maximum de tous les $\|d j(\mathcal{M}, V)\|$;

Si test $>\varepsilon$

- déplacement des nœuds dans la direction de $\operatorname{dj}(\mathcal{M}, V)$, (nouvelle triangulation $\left.\mathcal{T}_{h}^{(n)}\right)$;

- aller à Etape 1.

Sinon STOP.

Il faut préciser que les nœuds intérieurs du domaine peuvent se déplacer dans toutes les directions alors que ceux de la frontière peuvent se déplacer seulement sur la ligne de la frontière où ils se trouvent.

\section{Tests numériques :}

Les résultats numériques sont donnés à l'aide du logiciel FreeFem ++ v1.44 [7], pour $\Omega=[0,1] \times[0,1]$, un pas de maillage initial égale à $h=0.1$, et un $\varepsilon=10^{-4}$.

Exemple 1 : La fonction $F$ est la fonction caractéristique du cercle de centre $(1 / 2,1 / 2)$ et de rayon $1 / 4$.

On prend comme maillage initial $\mathcal{T}_{h}^{(0)}$ le maillage de pas $h=0.1$. La figure 1 (gauche) représente le maillage initial. A chaque itération de l'algorithme, les nouds se déplacent et la norme de l'estimateur hiérarchique diminue (voir Fig.3- à gauche). Dans la figure 1 ( droite), se trouve le maillage au bout de 584 itérations. Nous avons reporté dans la figure 3 (à droite), l'écart $\max \left(e_{h}\right)-\min \left(e_{h}\right)$. Nous remarquons qu'au file des itérations, cet écart diminue, ce qui signifie que l'erreur devient de plus en plus "équirépartie" (remarquons qu'il devient présque constant à partir de l'itération 200, et ceci pourrait constituer 
un critère pour arrêter les itérations de déplacement).

Exemple 2 : la solution exacte pour cet exemple est $u(x, y)=x y(1-x)(1-y)$.

Les mêmes remarques que l'exemple 1, peuvent être faites ici. Le maillage final (Fig.2à droite) est obtenu au bout de 143 itérations. Pour cet exemple, dans la pratique, il faut arrêter les itérations à 100 pour avoir un meilleur écart, car au delà de $100^{\text {me }}$ itération, l'écart augmente (voir Fig.4- à droite).

Conclusion : L'analyse décrite ici apporte une justification théorique au déplacement des nœuds qu'on utilisait auparavant d'une manière empirique. On peut dire aussi, que le processus de déplacement des nœuds conduit à une équidistributivité de l'erreur. Nous rappelons comme nous l'avons signalé en Introduction, que ce processus, est une des étapes dans un algorithme d'adaptation de maillage. Pour qu'il soit efficace, il faut l'accompagner avec d'autres opérations (raffinement, déraffinement, retournement d'arrêtes,...).

\section{Bibliographie}

[1] B. Achchab, A. Agouzal, J. Baranger, J.F. Maitre : « Estimateur d'erreur a posteriori hiérarchique : applications aux éléments finis mixtes », Numer. Math., 1998, 80, p. 159179.

[2] I. BABUSKA, W.C. RheinboldT : « Error estimates for adaptive finite elemement computation », SIAM Numer. Anal., 1978, 15, p.736-754.

[3] I. BAbuska, W.C. Rheinboldt : «A posteriri Error estimates for the finite elemement method », Int. J. Numer.Methh.engrg, 1978, 12, p 1597-1615 .

[4] R.E. BANK, A. WEISER : «Some a posteriori error estimator for elliptic partial differential equation », Math. Comp., 1985,p. 283-301,

[5] R.E. BANK, R.K. SMITH : «A posteriori error estimates based on hierarchical bases », SIAM J.Numer.Anal, 1993, Vol.30, Nº, p.921-935.

[6] M.C. Delfour, G. Payre, J.P. Zolesio : «An optimal triangulation for second order elliptic problems », Comp. Methods Appl. Mech. Engrg. 50, 1985, 231-261.

[7] F. Hecht, O. Pironneau, K. Ohtsuka : «FreeFem++ Manual », November 4, 2004.

[8] J. SoKolOWSKI, J.P. Zolesio : «Introduction to Optimization, shape sensitivity analysis » Springer series in computational mathematics, Vol.16, 1992.

[9] R. VERfURTH : «A reviw of a posteriori error estimation and adaptive Mesh-Refinement Techniques », Wiley and Tubner, 1996.

[10] J.P. Zolesio : «The Material- (or speed) Derivative methods for Shape Optimisation, in optimisation of distributed parameter structures » E.J.Haug and J.Cea (eds), Sijthof, and Noord- 
hoff, 1089-1151, (1981).

[11] J.P. ZOLESIO : « Les dérivée par rapport aux noeuds des triangularisations et leurs utilisations en identification des domaines », Ann. Sc. Math. Québec,1984, Vol.8, n 1, 97-120.
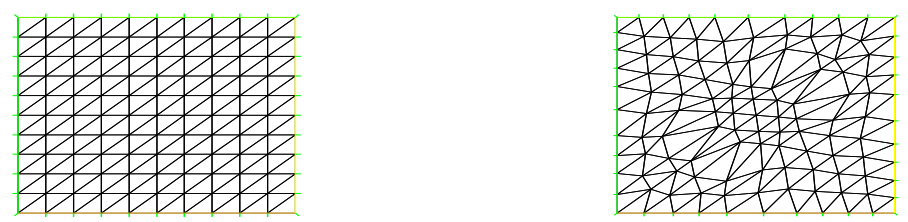

Figure 1. Maillage initial et maillage final (exemple 1).
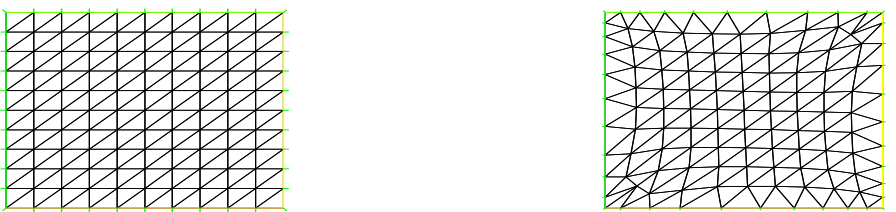

Figure 2. Maillage initial et maillage final (exemple 2). 

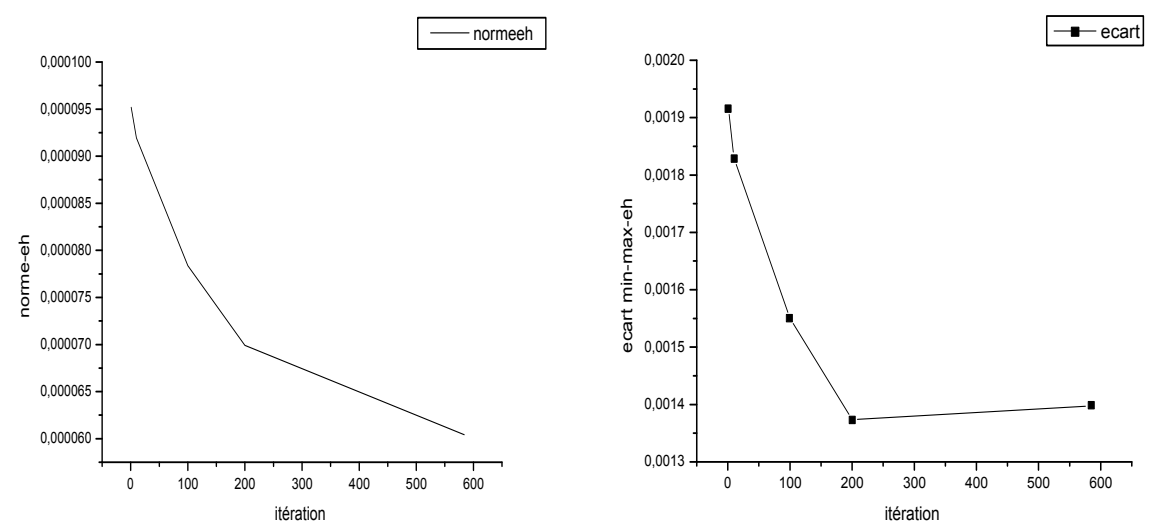

Figure 3. Norme de $e_{h}$ et l'écart $\max \left(e_{h}\right)-\min \left(e_{h}\right)$ (exemple 1).
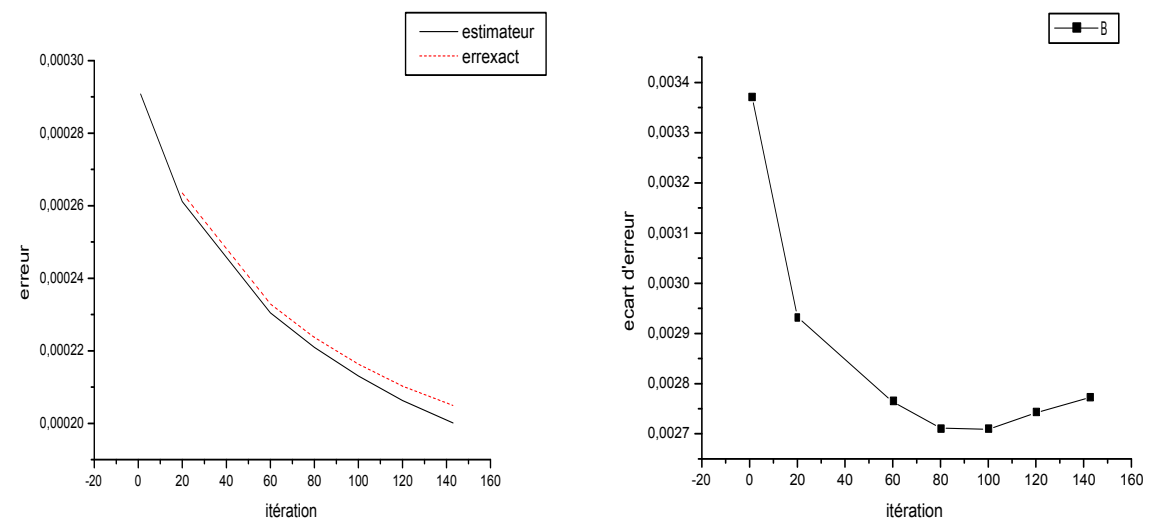

Figure 4. Norme de $e_{h}$ et l'écart $\max \left(e_{h}\right)-\min \left(e_{h}\right)$ (exemple 2). 